\title{
MODELACIÓN DEL TRANSPORTE DE PLAGUICIDAS E INSECTICIDAS EN SUELOS DE CULTIVO DE ARVEJA CON EL SOFTWARE HYDRUS-1D EN VEREDA EL ESCORIAL, PAMPLONA NORTE DE SANTANDER
}

\section{PESTICIDES AND INSECTICIDES TRANSPORTATION MODEL IN SOILS OF PEAS WITH HYDRUS-1D SOFTWARE IN VEREDA EL ESCORIAL, PAMPLONA NORTE DE SANTANDER}

\author{
Dayana Milena Moreno $\mathrm{P}^{1}$, María Esther Rivera ${ }^{2}$, Jesús R Delgado $\mathrm{R}^{3}$, Mario J Vargas $\mathrm{C}^{4}$ \\ ${ }^{1}$ Ingeniera, Universidad de Pamplona. Grupo de Investigaciones ambientales Agua, Aire y Suelo (GIAAS). \\ dayanamoreno063@gmail.com \\ ${ }^{2}$ Docente, Universidad de Pamplona. Grupo de Investigaciones ambientales Agua, Aire y Suelo (GIAAS). \\ ekaesther@gmail.com,jramondr@gmail.com,mvargas1594@hotmail.com
}

\section{Resumen}

En la presente investigación se modeló el transporte de plaguicidas e insecticidas en suelos de cultivo de arveja en el Escorial, Pamplona, mediante el software Hydrus 1-D, determinando compuestos químicos mediante cromatografía de gases con detector de captura de electrones y extracción por hidrocarburos aromáticos policíclicos. Se recopiló información bibliográfica, datos experimentales y de campo; observándose que las concentraciones a lo largo del perfil del suelo simulados del primer cultivo de Beta-BHC es $1.0 \mathrm{mg} / \mathrm{cm}^{3}$ en enero con profundidad hasta $39 \mathrm{~cm}$ en julio, GammaClordano con $0.38 \mathrm{mg} / \mathrm{cm}^{3}$ en febrero hasta $55 \mathrm{~cm}$ en julio, Endosulfan con $0.07 \mathrm{mg} / \mathrm{cm}^{3}$ en marzo hasta $59 \mathrm{~cm}$ en julio y Metoxicloro con 0.024 $\mathrm{mg} / \mathrm{cm}^{3}$ en marzo, $65 \mathrm{~cm}$ en septiembre. Para el segundo cultivo se observó que la concentración de Beta-BHC es $1.0 \mathrm{mg} / \mathrm{cm}^{3}$ en enero hasta $30 \mathrm{~cm}$ en mayo, Gamma-Clordano de $0.39 \mathrm{mg} / \mathrm{cm}^{3}$ en febrero hasta $40 \mathrm{~cm}$ en mayo, Endosulfan con $0.068 \mathrm{mg} / \mathrm{cm}^{3}$ en marzo hasta $50 \mathrm{~cm}$ en julio y Metoxicloro de $0.024 \mathrm{mg} / \mathrm{cm}^{3}$ en marzo hasta 59 $\mathrm{cm}$ en agosto. Se concluye que el Beta-BHC presentó mayor concentración en enero con 1.0 $\mathrm{mg} / \mathrm{cm}^{3}$ y el metoxicloro se transportó a mayor profundidad llegando hasta $65 \mathrm{~cm}$ para el primer cultivo y $59 \mathrm{~cm}$ para el segundo, indicando que sobrepasa los límites máximos permisibles.

Palabras clave: análisis arveja, contaminación.

\section{Abstract}

In the present investigation the transport of pesticides and insecticides was modeled in pea cultivation soils in El Escorial, Pamplona, using the Hydrus 1-D software, determining chemical compounds by means of gas chromatography with electron capture detector and extraction by aromatic hydrocarbons. polycyclic Bibliographic, experimental and field data were collected; observing that the concentrations along the simulated soil profile of the first Beta-BHC crop is $1.0 \mathrm{mg} / \mathrm{cm} 3$ in January with depth up to $39 \mathrm{~cm}$ in July, Gamma-Clordano with $0.38 \mathrm{mg} / \mathrm{cm} 3$ in February, up to $55 \mathrm{~cm}$ in July, Endosulfan with $0.07 \mathrm{mg} / \mathrm{cm} 3$ in March up to $59 \mathrm{~cm}$ in July and Methoxychlor with $0.024 \mathrm{mg} / \mathrm{cm} 3$ in March, 65 $\mathrm{cm}$ in September. For the second crop it was observed that the concentration of Beta-BHC is $1.0 \mathrm{mg} / \mathrm{cm} 3$ in January to $30 \mathrm{~cm}$ in May, Gamma-Clordano of $0.39 \mathrm{mg} / \mathrm{cm} 3$ in February to $40 \mathrm{~cm}$ in May, Endosulfan with $0.068 \mathrm{mg} / \mathrm{cm} 3$ in March up to $50 \mathrm{~cm}$ in July and Methoxychlor from $0.024 \mathrm{mg} / \mathrm{cm} 3$ in March to $59 \mathrm{~cm}$ in August. It is concluded that Beta-BHC presented higher concentration in January with $1.0 \mathrm{mg} / \mathrm{cm} 3$ and methoxychlor was transported deeper until reaching $65 \mathrm{~cm}$ for the first crop and $59 \mathrm{~cm}$ for the second, indicating that it exceeds the maximum permissible limits.

Keywords: analysis, peas, pollution. 
30

\section{Introducción}

En las últimas décadas por causa del crecimiento demográfico, se ha incrementado la actividad agrícola, requiriendo de una mayor producción para satisfacer las necesidades de la población. Desde entonces son evidentes los beneficios del uso de compuestos químicos (Ochoa, 2015) para prevenir, mitigar y controlar plagas de origen animal o vegetal en la agricultura ya que el resultado se percibe en el mantenimiento y aumento de las cosechas, pero a su vez el uso de estos compuestos permanecen inalterados por largo tiempo en el ambiente, debido a que son sustancias poco solubles en agua y terminan evaporándose en el aire o uniéndose a las partículas del suelo, causando así daños de gran magnitud al ambiente.

Como bien se sabe, la contaminación del suelo se puede deber a la presencia de sustancias químicas causadas por cualquier actividad humana y a partir de una cierta concentración se considera como no deseable, alterando la superficie terrestre, afectando gravemente al medio ambiente y generando efectos nocivos para los organismos del suelo, sus consumidores o inclusive transmitirse a otros sistemas (Sánchez, 2005).

De acuerdo con Pinzón, et al (2011), el empleo de plaguicidas de origen orgánico ha permitido obtener mejores calidades y mayores cosechas con respecto a la época en que se usaban solamente plaguicidas de origen inorgánico; sin embargo, los productos orgánicos son más difíciles de eliminar de los alimentos que las sales inorgánicas y por lo tanto han creado el problema potencial de residuos de plaguicidas altamente tóxicos para el ser humano y medio ambiente.

El uso de los plaguicidas e insecticidas en cultivos alteran el balance de la naturaleza, el cual desequilibra los sistemas ecológicos y a causa de esto producen varios fenómenos que probablemente afectan muchos de los elementos biológicos del suelo (Camazano, 1984). Por otro lado, a través de los años se han realizado diversas investigaciones sobre la simulación y modelación enfocadas al análisis de cultivos, en los cuales se han vertido agentes químicos con el fin de obtener una mejor producción, evitando la proliferación de plagas y enfermedades; mediante estos softwares se pueden modelar la contaminación del suelo y determinar el cambio de sus características físicas, químicas del suelo (Campos, 2013). y permiten realizar estudios e investigaciones que nos benefician para el futuro.

Por lo tanto, la presente investigación tuvo como fin modelar el transporte de plaguicidas e insecticidas en suelos de cultivo de arveja mediante el software HYDRUS-1D en la vereda el Escorial, municipio de Pamplona, Norte de Santander. La importancia de estudio radica en determinar las concentraciones de estos contaminantes presentes en el suelo y simular y modelar el transporte de estos mediante agentes químicos en el software en mención. De igual manera, aportar información a las entidades locales pertinentes sobre estudios de contaminación en el suelo producido por el uso de plaguicidas e insecticidas aplicados.

\section{Metodología}

Se procedió a modelar el transporte de los plaguicidas e insecticidas organoclorados encontrados en la cromatografía de los cultivos de arveja, cabe resaltar que para el suelo estándar no se realizó la modelación ya que es un suelo donde nunca ha sido cultivado, por ende no contiene ningún tipo de agente químico. Para la modelación se requirió:

\subsection{Recolección de información.}


31

Se recopilo información sobre diferentes estudiaos realizados a nivel internacional, nacional, regional y verificar la existencia de estudios a nivel local.

\subsection{Recolección de datos experimentales.}

La recolección de datos experimentales o datos obtenidos en otras investigaciones, son necesarios para realizar una calibración y validación del modelo usado por el software, dado que es el primer estudio a nivel local.

\subsection{Recolección de datos de campo.}

Se obtuvo la cantidad de material del suelo, propiedades hidráulicas y textura del suelo de cada uno de los cultivos propuestos para llevar a cabo la investigación (Tabla 1). Para ello, se realizó un apique de $100 \mathrm{~cm}$ de profundidad y donde se observó la textura y se midieron los horizontes del suelo, según Ortega, (2014).Tabla

1. Datos de campo para el software.

\begin{tabular}{|c|c|c|c|c|c|c|c|c|}
\hline \multicolumn{7}{|c|}{ Cultivo Arveja 1 Predio 1 } \\
\hline Muestra & Textura & Qr & Qs & $\begin{array}{c}\text { Alpha } \\
(\mathbf{1} / \mathbf{c m})\end{array}$ & $\mathbf{n}$ & $\begin{array}{c}\text { Ks } \\
\text { (cm/día) }\end{array}$ & I & Densidad \\
\hline 1 & $\begin{array}{c}\text { Arenoso } \\
\text { franco }\end{array}$ & 0,05 & 0,4 & 0,124 & 2,28 & 350,2 & 0,5 & 1,4 \\
\hline 2 & $\begin{array}{c}\text { Franco } \\
\text { arenoso }\end{array}$ & 0,06 & 0,4 & 0,075 & 1,89 & 106,1 & 0,5 & 1,6 \\
\hline \multicolumn{7}{|c|}{ Cultivo Arveja 2 Predio 2 } \\
\hline Muestra & Textura & Qr & Qs & $\begin{array}{c}\text { Alpha } \\
(\mathbf{1} / \mathbf{c m})\end{array}$ & $\mathbf{n}$ & $\begin{array}{c}\text { Ks } \\
\text { (cm/día) }\end{array}$ & I & Densidad \\
\hline 1 & $\begin{array}{c}\text { Franco } \\
\text { arenoso }\end{array}$ & 0,06 & 0,4 & 0,075 & 1,89 & 106,1 & 0,5 & 1,5 \\
\hline 2 & Arcilloso & 0,06 & 0,3 & 0,008 & 1,09 & 4,8 & 0,5 & 1,25 \\
\hline 3 & $\begin{array}{c}\text { Arcillo } \\
\text { limoso }\end{array}$ & 0,07 & 0,3 & 0,005 & 1,09 & 0,48 & 0,5 & 1,35 \\
\hline
\end{tabular}

Fuente: Hydrus 1D, Moreno (2017).

Así mismo, se datos precipitación y evaporación de 2016 suministrados por el IDEAM, se hallaron las densidades masivas dependiendo de la textura, los límites máximos permisibles, los coeficientes de distribución y la constante de degradación de cada uno de los contaminantes (Tabla 2) de acuerdo con Šimůnek, (1998).

Tabla 2. Parámetros de reacción de los contaminantes.

\begin{tabular}{|l|c|c|c|}
\hline Pesticidas & Kd & $\mathbf{m u}$ & $\begin{array}{c}\text { LMR } \\
\left(\mathbf{m g} / \mathbf{c m}^{\mathbf{3}}\right)\end{array}$ \\
\hline Beta - BHC & 0,368 & 0,075 & 0.00003 \\
\hline Gamma - Clordano & 0,278 & 0,07 & 0.00025 \\
\hline Endosulfan & 0,475 & 0,11 & 0.00004 \\
\hline Metoxicloro & 0,468 & 0,03 & 0.0005 \\
\hline
\end{tabular}

Fuente: Torres (2008), EPA (2016)

\section{Resultados}

En la figura 1 se observa la infiltración potencial en superficie que se genera para el cultivo de arveja 1 a lo largo de un año, es decir, la infiltración potencial para todo un año, podría alcanzar valores de $4.1 \mathrm{~cm} / \mathrm{dia}$ en el mes de mayo, $2.1 \mathrm{~cm} /$ dia en el mes de abril, $1.8 \mathrm{~cm} /$ dia en el mes de agosto y $2.5 \mathrm{~cm} /$ dia en el mes de octubre.

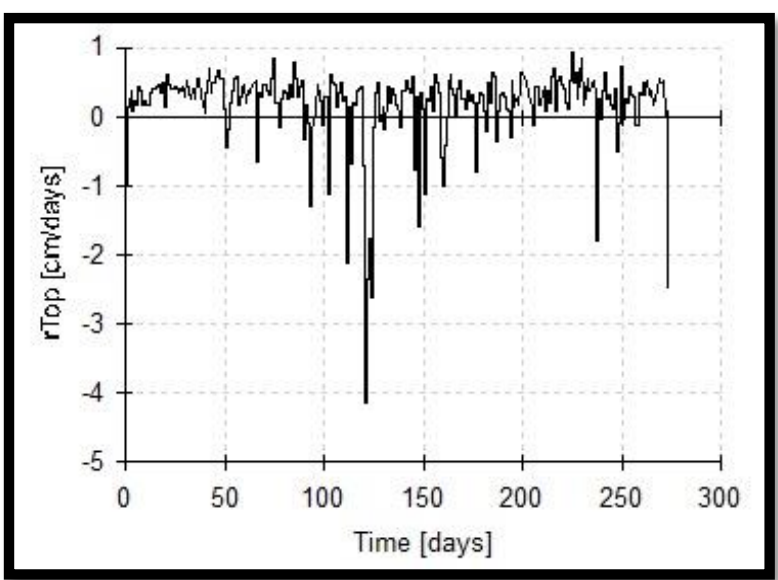

Figura 1. Infiltración potencial en superficie del cultivo de arveja 1

En la figura 2 se aprecia la modelación del transporte del contaminante Beta- BHC en el suelo del cultivo de arveja 1. Esta concentración va aumentando desde el mes de mayo con un nivel de $0.00009 \mathrm{mg} / \mathrm{cm}^{3}$ hasta el mes de junio con $0.00029 \mathrm{mg} / \mathrm{cm}^{3}$ y empieza a disminuir hasta el mes de septiembre con concentración de 0.00010 
32

$\mathrm{mg} / \mathrm{cm}^{3}$. Así mismo se observa las concentraciones a lo largo del perfil del suelo en los 366 días simulados, donde la concentración de Beta-BHC comienza con $1.0 \mathrm{mg} / \mathrm{cm}^{3}$ y disminuye

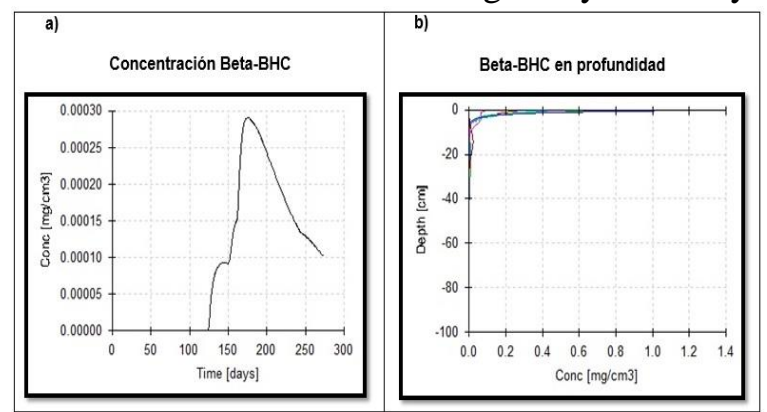

en profundidad hasta 20 y $30 \mathrm{~cm}$, lo que indica que no llegaría a contaminar las aguas subterráneas.

Figura 2. Curvas de transporte del contaminante Beta-BHC en cultivo arveja 1

De la figura 3-a, se observa que la concentración de Gamma-Clordano va aumentando desde el mes de mayo con un nivel de $0.0011 \mathrm{mg} / \mathrm{cm} 3$ hasta el mes de junio con $0.0024 \mathrm{mg} / \mathrm{cm} 3$ y empieza a disminuir hasta el mes de octubre con concentración de $0.00075 \mathrm{mg} / \mathrm{cm} 3$. De la figura 3-b la concentración de Gamma-Clordano

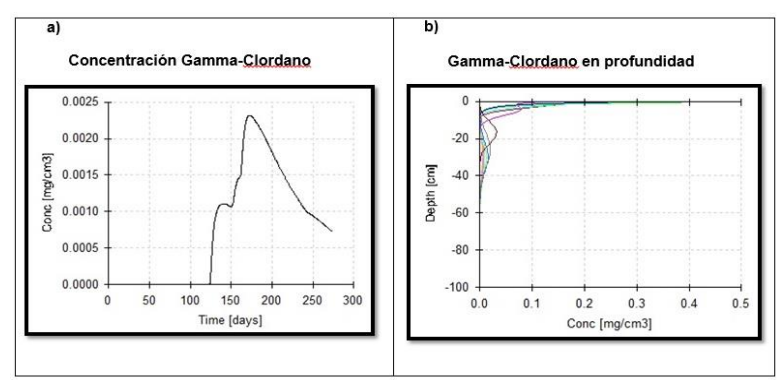

comienza con $0.38 \mathrm{mg} / \mathrm{cm} 3$ y disminuye en profundidad hasta $55 \mathrm{~cm}$, es decir que tampoco alcanza a contaminar las aguas subterráneas

Figura 3. Curvas de transporte del contaminante Gamma-Clordano en arveja 1.

De la figura 4-a, se determina que la concentración de Endosulfan comienza a aumentar desde el mes de mayo hasta el mes de julio con $0.0014 \mathrm{mg} / \mathrm{cm} 3$ en superficie y empieza a disminuir hasta llegar al mes de octubre con una concentración de $0.00089 \mathrm{mg} / \mathrm{cm} 3$. De la figura 4-b la concentración de Endosulfan aumenta más en la superficie del suelo con $0.07 \mathrm{mg} / \mathrm{cm} 3 \mathrm{y}$ disminuye en profundidad hasta $59 \mathrm{~cm}$, indicando

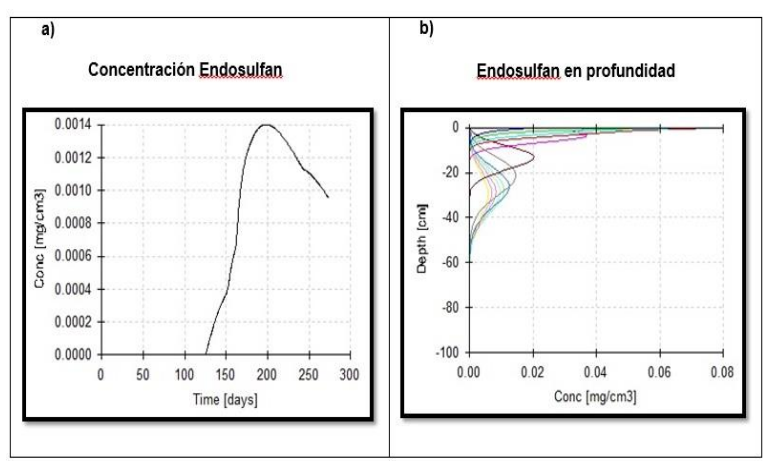

que la alta infiltración potencial registrada en el mes de mayo hace que este soluto pueda alcanzar este valor.

Figura 4. Curvas de transporte del contaminante Endosulfan en arveja 1.

De la figura 5-a, se observa que la concentración de Metoxicloro aumenta desde el mes de mayo hasta el mes de septiembre con un nivel de 0.0030 $\mathrm{mg} / \mathrm{cm} 3$ en superficie y en la figura 5-b la concentración de Metoxicloro aumenta en el tiempo debido al constante vertimiento, reportando cantidades $0.024 \mathrm{mg} / \mathrm{cm} 3 \quad \mathrm{y}$ disminuyendo en profundidad hasta $65 \mathrm{~cm}$, indicando que la alta infiltración potencial registrada en el mes de mayo hace que este soluto pueda alcanzar este valor. Es decir que el principal factor para el transporte de cualquier soluto depende principalmente de las propiedades físicas y químicas del suelo, a su vez, según la modelación, el constante vertimiento de los

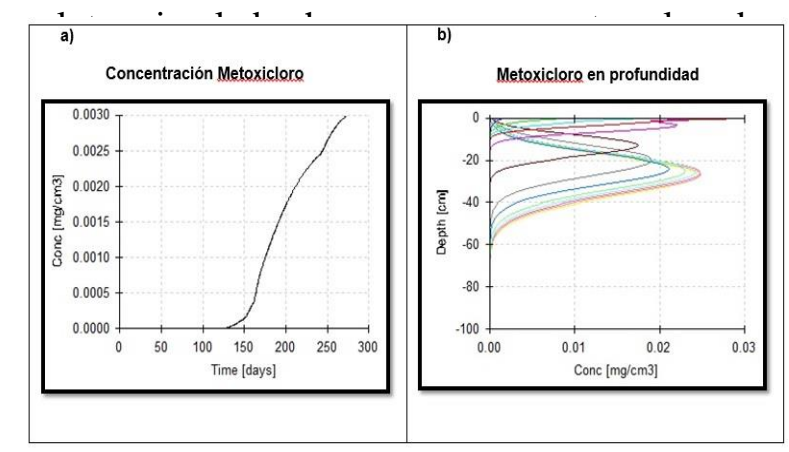

Figura 5. Curvas de transporte del contaminante Metoxicloro en arveja 1 
En la figura 6 se observa la infiltración potencial en superficie para el cultivo de arveja 2 a lo largo de un año, alcanzando un mayor valor de 4.1 $\mathrm{cm} /$ dia en el mes de mayo, seguido por valores de $2.1 \mathrm{~cm} /$ dia en el mes de abril, $1.8 \mathrm{~cm} /$ dia en el mes

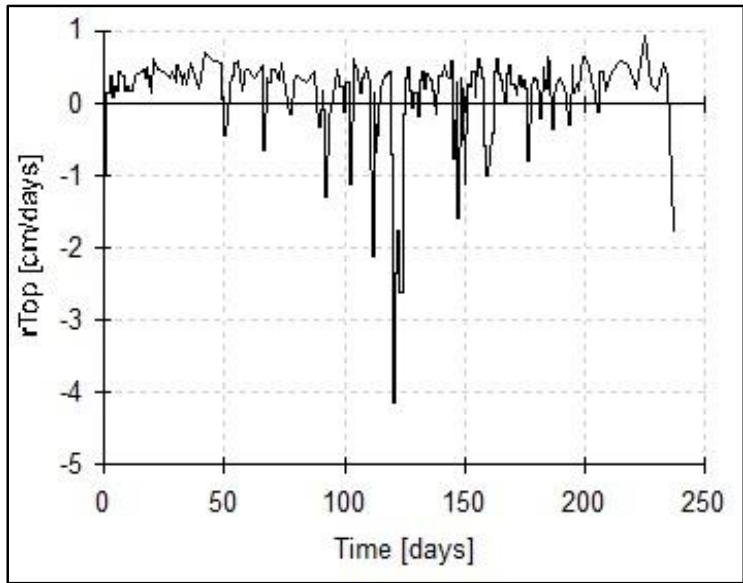

de mayo y $1.9 \mathrm{~cm} /$ dia en el mes de agosto; teniendo similitud con la infiltración potencial del cultivo de arveja 1.

Figura 6. Infiltración potencial en superficie del cultivo de arveja 2

En la figura 7-a, se aprecia que la concentración de Beta-BHC aumenta desde el mes de mayo hasta julio con un nivel de $0.000013 \mathrm{mg} / \mathrm{cm} 3 \mathrm{y}$ empieza a disminuir hasta el mes de agosto con concentración de $0.0000079 \mathrm{mg} / \mathrm{cm}^{3}$. De la figura 7-b la concentración de Beta-BHC comienza con

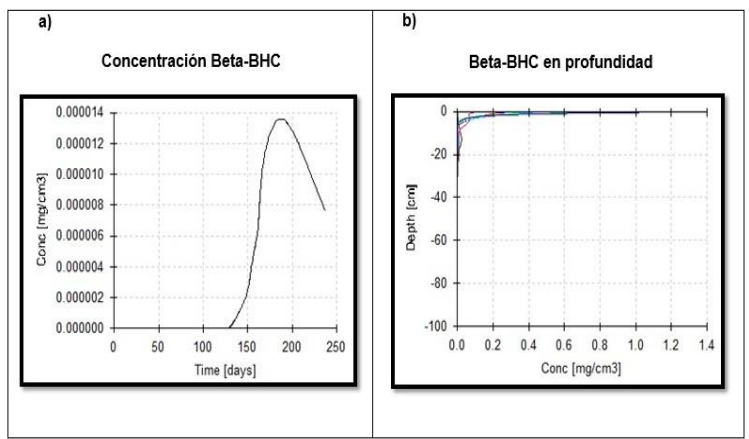

$1.0 \mathrm{mg} / \mathrm{cm}^{3}$ y disminuye en profundidad hasta 20 y $30 \mathrm{~cm}$, indicando que es similar al cultivo de arveja 1, sus resultados dependen de las características físicas y químicas del suelo.

Figura 7. Curvas de transporte del contaminante Beta-BHC en arveja 2.
De la figura 8-a, se observa que la concentración de Gamma-Clordano comienza a aumentar desde el mes de mayo hasta julio con un nivel de $0.00011 \mathrm{mg} / \mathrm{cm} 3$ y empieza a disminuir hasta el mes de agosto con concentración de 0.00006 $\mathrm{mg} / \mathrm{cm} 3$. De la misma manera para la figura $8-b$

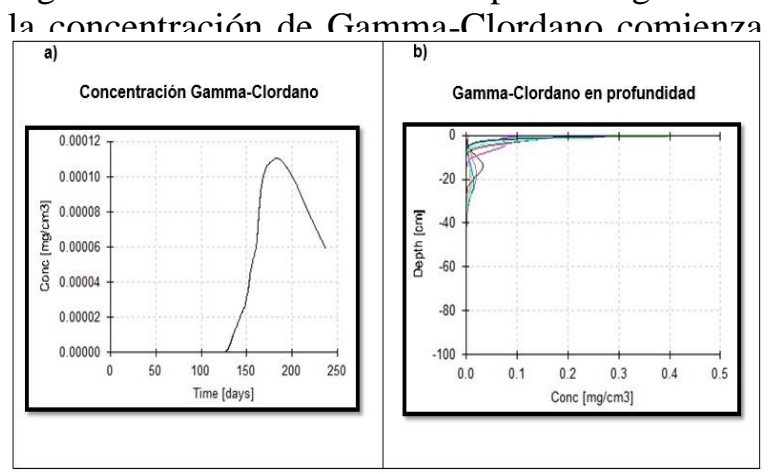

De la figura 9-a, se percibe que la concentración de Endosulfan aumenta desde el mes de mayo hasta el mes de julio con $0.00011 \mathrm{mg} / \mathrm{cm}^{3}$ en superficie y empieza a disminuir hasta llegar al mes de agosto con una concentración de 0.000081 $\mathrm{mg} / \mathrm{cm}^{3}$. De la figura 9-b la concentración de Endosulfan aumenta en el tiempo debido al vertimiento con $0.068 \mathrm{mg} / \mathrm{cm} 3$ y disminuye en profundidad hasta $50 \mathrm{~cm}$, indicando que la alta infiltración potencial registrada en el mes de mayo hace que este soluto pueda alcanzar este valor, de acuerdo con Valderrama, (2015).

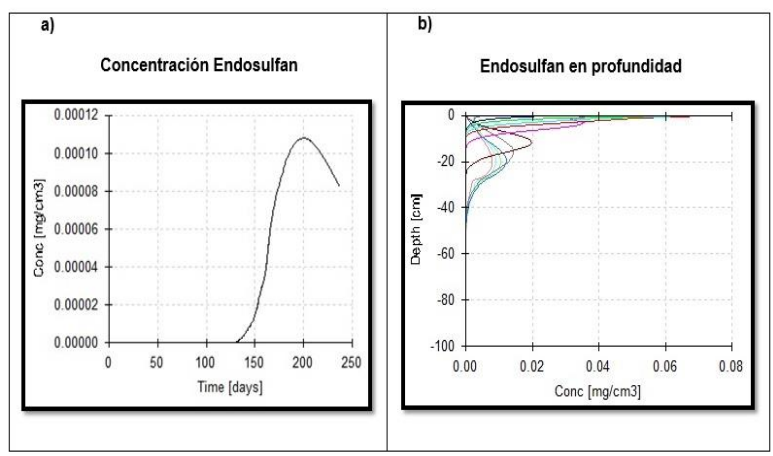

Figura 9. Curvas de transporte del contaminante Endosulfan en arveja 2. 
es el que mayor profundidad se transportó

34

De la figura 10-a, se infiere que la concentración de Metoxicloro aumenta desde el mes de mayo hasta el mes de agosto con un nivel de 0.00051 $\mathrm{mg} / \mathrm{cm} 3$ en superficie y de la figura $10-\mathrm{b}$ la concentración de Metoxicloro aumenta en el \begin{tabular}{|ll|l|l|l|l|}
\hline a) & & & \\
\hline
\end{tabular}

Figura 10. Curvas de transporte del contaminante Metoxicloro en arveja

Para el cultivo de arveja 1 y 2 presentó mayor transporte el contaminante metoxicloro en el suelo, es importante tener en cuenta la cantidad de material de los dos cultivos y la textura del suelo, para el primer cultivo se trabajó con 2 materiales, es por ello que el contaminante se transportó hasta $65 \mathrm{~cm}$ de profundidad, a comparación del segundo cultivo que presentaba 3 materiales y se transportó $59 \mathrm{~cm}$ de profundidad según lo dicho

\section{Conclusiones}

Los contaminantes en los suelos de los cultivos de arveja son plaguicidas e insecticidas organoclorados tales como el Beta BHC, Gamma clordano, Endosulfan y Metoxicloro.

En la modelación del transporte de los contaminantes en el suelo se determinó que para los dos cultivos, el lindano es el que mayor concentración presentó para el mes de enero con $1.0 \mathrm{mg} / \mathrm{cm}^{3}$ en los dos cultivos y el Metoxicloro llegando hasta $65 \mathrm{~cm}$ para el primer cultivo y 59 $\mathrm{cm}$ para el segundo.

\section{Agradecimientos}

A la universidad de Pamplona por facilitar los recursos humanos como la Auxiliar Yolanda Rico y PhD. Alfonso Quijano Parra, laboratorio de Control de calidad y de diagnóstico, Laboratorio de suelo, para la realización de este proyecto.

\section{Referencias Bibliografícas}

Campos, D. (2013). SlideShare. Obtenido de Propiedades fisicas de los suelos: https://es.slideshare.net/vandick20/propi edades-fsica-de-los-suelos

Maldonado, J. (2012). Características físicoquímicas de los plaguicidas. En http://www.crc.gov.co/files/Respel/Pl aguicidas.pdf

Ochoa, R. (2015). SlideShare. Obtenido de Riesgos y beneficios de los plaguicidas: En

https://es.slideshare.net/ricardoochoa 9404/cuales-son-los-riesgos-ybeneficios-de-los-plaguicidas-ypesticidas

Ortega, G. (2014). Composición y clasificación de los suelos. En http://www.abc.com.py/edicionimpresa/suplementos/escolar/compos icion-y-clasificacion-de-los-suelos$\underline{1293271 . h t m l}$

1

Pinzón MI, Londoño A, Blach D, Gutiérrez JA, Rojas A M. Determinación de residuos de 
plaguicidas organoclorados POR gc$\mu E C D$ en frutos de PIÑA (Ananas comosus L.) variedad Golden MD2 en el departamento del Quindío. Bistua $.2011 ; 9(2): 4-8$

Sánchez, M. (2005). Contamianción de suelos y técnicas de remediación. En https://es.slideshare.net/MarcoAntoni oArribasp/contaminacion-de-suelos2017pptx-y-las-tecnicas-deremediacin

Šimůnek, (1998). HYDRUS-1D, Agua Subterranea Modelacion Centro Internacional. Colorado.

Valderrama, J. (2015). Persistencia de plaguicidas en el ambiente y su ecotoxicidad. En http://www.academia.edu/11377 488/TRANSPORTE Y TRANSFORM ACION DE PLAGUICIDAS EN EL M $\underline{\text { EDIO }}$

D.M. Moreno P, Ing. Ambiental (2018) de la Universidad de Pamplona - Miembro activo del Semillero de Investigación Ambiental de Agua, Aire y Suelo (SIAAS).

M.E. Rivera, Lic. En Matemáticas y Física (1993) de la Universidad Francisco de Paula Santander - UFPS, doctorado en Hidrología (2003) de la Universidad Estatal Hidrometeorológica de San Petersburgo, Rusia. Profesor titular de la Universidad de Pamplona, Colombia. Miembro activo del Grupo de Investigación Ambiental de Agua, Aire y Suelos (GIAAS). Coordinador del Semillero de Investigaciones Ambientales de Agua, Aire y Suelo (SIAAS). Dirige la línea de modelación y simulación de recursos hídricos. Los intereses de investigación incluyen: simulación, modelado, estadísticas, diseño experimental, gestión y conservación de cuencas hidrológicas. Desde 2007 trabajó con diseño experimental en Maestría en Ciencia y Tecnología de alimentos y Maestría en Química y en Ing. Ambiental imparte Hidrología. ORCID: 0000-0002-1289-9776.

J.R. Delgado Rodríguez, Geólogo (1994) de la Universidad Industrial de Santander - UIS; Magister en Geotecnia (2001) de la Universidad Nacional de Colombia, Bogotá, DC. Profesor Asociado de la Universidad de Pamplona, Facultad de Ingenierías y Arquitectura; Integrante activo del Grupo de Investigaciones Ambientales agua, aire y suelo (GIAAS), Miembro de la Sociedad Colombiana de Geotecnia; Consultor en Geología, Geotecnia y cimentaciones para obras viales. http:// orcid.org/0000-0002-3397

*Para citar este artículo: Moreno P DM,Rivera ME, Delgado R JR, Vargas C MJ.. PESTICIDES AND INSECTICIDES TRANSPORTATION MODEL IN SOILS OF PEAS WITH HYDRUS-1D SOFTWARE IN VEREDA EL ESCORIAL, PAMPLONA NORTE DE SANTANDER. Revista Bistua.2017.15(2)29-35

+ Autor para el envió de correspondencia y la solicitud de las separatas: Moreno P DM. Ingeniera, Universidad de Pamplona. Grupo de Investigaciones ambientales Agua, Aire y Suelo (GIAAS). dayanamoreno063@gmail.com

Recibido: Octubre 18 de 2016

Aceptado: Enero 28 de 2017 
